DOI: $10.1007 / \mathrm{s} 00209-005-0847-0$

\title{
Minimal weakly isotropic forms
}

\author{
Karim Johannes Becher \\ École Polytechnique Fédérale de Lausanne, SB IMB CSAG, MA C3 595, Station 8, \\ CH - 1015 Lausanne, Switzerland (e-mail: becher@maths .ucd.ie)
}

Received: 27 September 2004; in final form: 21 March 2005 /

Published online: 16 August 2005 - (C) Springer-Verlag 2005

\begin{abstract}
In this article weakly isotropic quadratic forms over a (formally) real field are studied. Conditions on the field are given which imply that every weakly isotropic form over that field has a weakly isotropic subform of small dimension. Fields over which every quadratic form can be decomposed into an orthogonal sum of a strongly anisotropic form and a torsion form are characterized in different ways.
\end{abstract}

Mathematics Subject Classification (2000): 11E04, 11E81, 12D15

\section{Introduction}

The concept of weak isotropy was introduced by Prestel in [15] and studied in the 1970ies, especially by Prestel and Bröcker. The main motivation for this concept is the failure of any kind of local-global principle for isotropy of quadratic forms in a general situation. It turned out that, over any (formally) real field, weakly isotropic forms can be characterized, on the one hand in terms of the semi-orderings (cf. [15]), on the other hand in terms of the real places of the field (cf. [15], [4]). Applications of weakly isotropic forms occur in real algebra and geometry (cf. [17]).

The present article investigates weakly isotropic forms in view of the question what kind of subforms and orthogonal decompositions they admit. This leads to consider in particular those weakly isotropic forms which are minimal in the sense that they do not contain any proper subform which is weakly isotropic. The question of how large the dimension of such a form may be gives rise to a new field invariant. This invariant will be compared with other invariants and properties of fields. This work is mainly inspired by ideas and results due to Arason and Pfister, found in [1].

The reader is assumed to be familiar with the general theory of quadratic forms over fields as it is presented for example in the books by Lam and Scharlau (cf. 
[9] or [18]). The terms 'form' and 'quadratic form' shall always refer to a regular, finite dimensional quadratic form.

Let $F$ always be a field of characteristic different from 2. A quadratic form over $F$ may be given by a diagonalization $\left\langle a_{1}, \ldots, a_{n}\right\rangle$ with entries $a_{1}, \ldots, a_{n} \in F^{\times}$ which determines the form up to isometry. We denote by $W F$ the Witt ring of (Witt equivalence classes of) quadratic forms over $F$ and by $I F$ the fundamental ideal in $W F$, given by the even-dimensional forms. For $n \geq 1$ we write $I^{n} F$ for the $n$th power $(I F)^{n}$ of the ideal $I F$.

A quadratic form $\varphi$ over $F$ is said to be weakly isotropic if there is an integer $m \geq 1$ such that $m \times \varphi=\varphi \perp \ldots \perp \varphi$ is isotropic; the lowest such $m$ shall then be called the weak isotropy index of $\varphi$ and denoted by $w i(\varphi)$. Note that $w i(\varphi)=1$ means that $\varphi$ is isotropic. We say that $\varphi$ is strongly anisotropic if it is not weakly isotropic and write $w i(\varphi)=\infty$ in this case.

In order to study weakly isotropic forms one needs also to consider torsion forms. A quadratic form $\varphi$ over $F$ is torsion if $m \times \varphi$ is hyperbolic for a certain $m \geq 1$. The least such $m$ then is a power of 2 (cf. [18, p. 53]) and equal to the order of the class of $\varphi$ in $W F$. By Pfister's local-global principle (cf. [13, Satz 22]), a quadratic form over $F$ is torsion if and only if its signature at every ordering of $F$ is zero.

We denote by $F^{\times}$the multiplicative group of the field $F$ and by $\sum F^{\times 2}$ the subgroup consisting of the non-zero sums of squares in $F$. The elements of $\sum F^{\times 2}$ are said to be totally positive. If $-1 \in \sum F^{\times 2}$, then the field $F$ is said to be nonreal, otherwise $F$ is real. If $F$ is a nonreal field, then every quadratic form over $F$ is torsion and in particular weakly isotropic. The notion of weak isotropy is therefore only interesting over real fields.

Let $\beta$ be a binary (i.e. 2-dimensional) quadratic form over $F$. Writing $\beta=$ $\langle a,-a d\rangle$ with $a, d \in F^{\times}$, we have that $\beta$ is weakly isotropic if and only if $\beta$ is torsion, if and only if $d \in \sum F^{\times 2}$. If a quadratic form contains a torsion binary form, then it is clearly weakly isotropic. The question whether the converse implication also holds is the starting point for the present investigation. Given a weakly isotropic quadratic form $\varphi$ over a real field $F$, does $\varphi$ contain a torsion binary form?

If the answer to this question is positive for every weakly isotropic form over $F$, then it follows by an induction argument that every quadratic form over $F$ has a 'weak decomposition' in the following sense. If a quadratic form $\varphi$ over a real field $F$ can be decomposed into an orthogonal sum

$$
\gamma \perp \beta_{1} \perp \ldots \perp \beta_{r}
$$

where $\gamma$ is a strongly anisotropic form and $\beta_{1}, \ldots, \beta_{r}(r \geq 0)$ are torsion binary forms over $F$, then this is called a weak decomposition of $\varphi$. Note that the number $r$ then is uniquely determined by $\varphi$, and so is the isometry class of $\gamma_{F_{p y t h}}$ over the pythagorean closure $F_{\text {pyth }}$ of $F$.

We say that $F$ has the weak decomposition property or that $F$ is a WD-field, if either $F$ is nonreal, or if every quadratic form over $F$ has a weak decomposition in the above sense.

In (4.2) we will characterize real WD-fields as those fields having the Property $\mathrm{C}$ investigated by Arason and Pfister in [1]. Property $\mathrm{C}$ means that torsion forms are 
'strongly balanced', i.e. decomposable into a sum of binary torsion forms. Easily accessible examples of $W D$-fields are number fields and extensions of transcendence degree at most two of real closed fields.

Arason and Pfister have also obtained examples of real fields not satisfying Property C. In [1, Satz $2 \&$ Satz 3] they show that if $k$ is a real field with two totally positive elements $u, v$ such that $v$ is not represented by $\langle 1, u\rangle$, then the 3-dimensional form $\left\langle 1, X,-\left(u+Y^{2}+v X\right)\right\rangle$ over the field $k(X, Y)$ is weakly isotropic but contains no torsion binary form. In particular the form $\left\langle 1, X,-\left(1+Y^{2}+3 X\right)\right\rangle$ over the field $\mathbb{Q}(X, Y)$ gives evidence that this is not a $W D$-field.

This example suggests to study the following concepts. A weakly isotropic quadratic form is said to be minimal weakly isotropic (m.w.i.) if none of its proper subforms is weakly isotropic. Since it is desirable to have an upper bound for the dimensions of minimal weakly isotropic forms over $F$, we define

$$
w(F)=\sup \{\operatorname{dim}(\varphi) \mid \varphi \text { m.w.i. form over } F\} \quad \in \mathbb{N} \cup\{\infty\} .
$$

We consider $w(F)$ as an invariant of the field $F$ and want to compare it with other field invariants related to quadratic form theory. For a nonreal field $F$ it seems natural to agree that $w(F)=1$. If $F$ is real, then the hyperbolic plane $\mathbb{H}=\langle 1,-1\rangle$ should be considered as a minimal weakly isotropic form so that we obtain $w(F) \geq 2$ in this case. With these definitions, $F$ is a $W D$-field if and only if $w(F) \leq 2$.

The Pythagoras number of $F$ is denoted by $p(F)$; this invariant is defined as the least integer $m \geq 1$ such that every totally positive element in $F$ is equal to a sum of $m$ squares if such an integer exists, otherwise $p(F)=\infty$.

The following section provides some preliminary results, in particular on products of two quadratic forms where one of them is multiplicative or represents only elements which are sums of squares in the field.

In Section 3 we obtain two upper bounds on the invariant $w(F)$. On the one hand, $w(F) \leq p(F)$ holds for any field $F$ which is not real pythagorean (3.3). On the other hand, if $n \geq 3$ is such that $I^{n} F$ is torsion-free, then $w(F) \leq 2^{n-2}(3.5)$. The latter also allows us to compare $w(F)$ with the $u$-invariant of the field $F$ (3.6).

In Section 4 we show that for $m \geq 3$ it suffices that there exists no minimal weakly isotropic form of dimension $m$ over $F$ in order to have $w(F)<m$ (4.1). This criterion allows us in (4.2) to characterize $W D$-fields in a way that extends $[1$, Satz 4]. A list of examples of $W D$-fields is given in (4.3).

In Section 5 we introduce three further field invariants $b, b^{\prime}$, and $p u$ encoding properties of fields related to sums of squares. For a non-pythagorean field $F$, we refine the previously shown inequality $w(F) \leq p(F)$ to a chain of inequalities $w(F) \leq b(F) \leq b^{\prime}(F) \leq p u(F) \leq p(F)$. Furthermore, some open problems are formulated.

\section{Totally positive quadratic forms}

For any quadratic form $\varphi$ over $F$ we denote by $D_{F}(\varphi)$ the set of non-zero elements of $F$ which are represented by $\varphi$. Recall that the form $\varphi$ over $F$ is said to be $m u l$ tiplicative if either $\varphi$ is hyperbolic or if $\varphi$ is anisotropic and $a \varphi \cong \varphi$ holds for 
any $a \in D_{F}(\varphi)$. For $a_{1}, \ldots, a_{n} \in F^{\times}$the form $\left\langle 1,-a_{1}\right\rangle \otimes \cdots \otimes\left\langle 1,-a_{n}\right\rangle$ over $F$ is called an $n$-fold Pfister form and denoted by $\left\langle\left\langle a_{1}, \ldots, a_{n}\right\rangle\right\rangle$. Pfister forms are multiplicative. In particular any Pfister form is either hyperbolic or anisotropic.

Proposition 2.1. Let $\rho$ be a multiplicative form and $\varphi$ an arbitrary quadratic form over $F$.

(a) Assume that $\operatorname{dim}(\varphi) \geq 2$ and let $a_{1}, \ldots, a_{n} \in F^{\times}$be such that $\varphi \cong\left\langle a_{1}, \ldots, a_{n}\right\rangle$. The form $\rho \otimes \varphi$ is isotropic over $F$ if and only if there exist $t_{1}, \ldots, t_{n} \in D_{F}(\rho)$ such that $\left\langle a_{1} t_{1}, \ldots, a_{n} t_{n}\right\rangle$ is isotropic. Moreover, the choice of $t_{1}, \ldots, t_{n}$ can be made such that $t_{1}=1$.

(b) If $\rho \otimes \varphi$ is hyperbolic and $\psi$ is a subform of $\varphi$ with $\operatorname{dim}(\psi)>\frac{1}{2} \operatorname{dim}(\varphi)$, then $\rho \otimes \psi$ is isotropic.

(c) If $\operatorname{dim}(\varphi) \geq 3$ and if $\rho \otimes \varphi$ is isotropic, then there exists a form $\vartheta \in I^{2} F$ with $\operatorname{dim}(\vartheta)=2 \operatorname{dim}(\varphi)-2$ such that $\varphi$ is a subform of $\vartheta$ and such that $\rho \otimes \vartheta$ is hyperbolic.

Proof. If $\rho$ is hyperbolic then all statements are trivial. We may thus assume that the multiplicative form $\rho$ is anisotropic. Statement (a) is well-known (cf. [10, Chap. 1]). Statement (b) is obvious, since $\rho \otimes \psi$ is a subform of $\rho \otimes \varphi$ with $\operatorname{dim}(\rho \otimes \psi)>$ $\frac{1}{2} \operatorname{dim}(\rho \otimes \varphi)$. For the proof of (c), suppose that $\rho \otimes \varphi$ is isotropic. Let $a_{1}, \ldots, a_{n} \in$ $F^{\times}$be such that $\varphi \cong\left\langle a_{1}, \ldots, a_{n}\right\rangle$. We assume that $n=\operatorname{dim}(\varphi) \geq 3$. By (a), there exist $t_{1}, \ldots, t_{n} \in D_{F}(\rho)$ such that $\left\langle a_{1} t_{1}, \ldots, a_{n} t_{n}\right\rangle$ is isotropic, hence of the form $\psi^{\prime} \perp\langle c, 1,-1\rangle$ for a certain $c \in F^{\times}$and some form $\psi^{\prime}$ over $F$, where $\operatorname{dim}\left(\psi^{\prime}\right)=$ $(n-3) \geq 0$. Let $t=t_{1} \cdots t_{n}$. Since $\rho$ is multiplicative we have $t \in D_{F}(\rho)$. We put $\psi=\psi^{\prime} \perp\langle c t\rangle$ and $\vartheta=\varphi \perp-\psi$. Note that $\operatorname{dim}(\vartheta)=2 \operatorname{dim}(\varphi)-2$. Now $\vartheta$ is Witt equivalent to the form $a_{1}\left\langle 1,-t_{1}\right\rangle \perp \cdots \perp a_{n}\left\langle 1,-t_{n}\right\rangle \perp c\langle 1,-t\rangle$. Therefore $\vartheta \in I^{2} F$ and $\rho \otimes \vartheta$ is hyperbolic, as $\rho$ is multiplicative and represents $t_{1}, \ldots, t_{n}$ and $t$.

We are now going to apply the proposition to link weakly isotropic forms and torsion forms with each other.

Corollary 2.2. Let $\varphi$ be a quadratic form over $F$.

(a) Assume that $\operatorname{dim}(\varphi) \geq 2$ and let $a_{1}, \ldots, a_{n} \in F^{\times}$be such that $\varphi \cong\left\langle a_{1}, \ldots, a_{n}\right\rangle$. Then $\varphi$ is weakly isotropic over $F$ if and only if there exist $t_{1}, \ldots, t_{n} \in \sum F^{\times 2}$ such that the form $\left\langle a_{1} t_{1}, \ldots, a_{n} t_{n}\right\rangle$ is isotropic. Moreover, the choice of $t_{1}, \ldots, t_{n}$ can be made such that $t_{1}=1$.

(b) If $\varphi$ is torsion (of order $2^{m}$, with $m \geq 0$ ) and if $\psi$ is a subform of $\varphi$ such that $\operatorname{dim}(\psi)>\frac{1}{2} \operatorname{dim}(\varphi)$, then $\psi$ is weakly isotropic (of index wi $(\varphi) \leq 2^{m}$ ).

(c) If $\operatorname{dim}(\varphi) \geq 3$ and if $\varphi$ is weakly isotropic, then there exists a torsion form $\vartheta \in I^{2} F$ with $\operatorname{dim}(\vartheta)=2 \operatorname{dim}(\varphi)-2$ and such that $\varphi$ is a subform of $\vartheta$.

Proof. For $m \geq 0$ we put $\rho_{m}=2^{m} \times\langle 1\rangle=\langle\langle-1, \ldots,-1\rangle\rangle$ and observe that this form is multiplicative. A form $\varphi$ is weakly isotropic (resp. torsion) if and only if $\rho_{m} \otimes \varphi$ is isotropic (resp. hyperbolic) for $m$ sufficiently large. Now the statements follow immediately from the proposition. 
Remark 2.3. Let $T$ be a preordering of $F$, that is, a subset of $F$ which is closed under addition and under multiplication, and which contains all squares in $F$. A quadratic form $\varphi$ over $F$ is said to be $T$-isotropic (resp. $T$-hyperbolic) if there exist $t_{1}, \ldots, t_{m} \in T \backslash\{0\}$ such that $\left\langle t_{1}, \ldots, t_{m}\right\rangle \otimes \varphi$ is isotropic (resp. hyperbolic). It is easily observed that all statements of the corollary remain valid if 'weakly isotropic' and 'torsion' are replaced by ' $T$-isotropic' and ' $T$-hyperbolic', respectively, and if $\sum F^{\times 2}$ is replaced by $T^{\times}=T \backslash\{0\}$ in (a). The proof then uses the fact that a form $\varphi$ over $F$ is $T$-isotropic (resp. $T$-hyperbolic) if and only if $\left\langle\left\langle-t_{1}, \ldots,-t_{m}\right\rangle\right\rangle \otimes \varphi$ is isotropic for some non-zero elements $t_{1}, \ldots, t_{n} \in T$. For our purposes, we only need the special case where $T=\sum F^{\times 2} \cup\{0\}$, which is formulated in the corollary.

We say that a form $\rho$ over $F$ is totally positive if $D_{F}(\rho) \subset \sum F^{\times 2}$. If we have equality $D_{F}(\rho)=\sum F^{\times 2}$, then we say that $\rho$ is positive-universal. A typical example of a positive-universal form over a real field $F$ is obviously the form $p \times\langle 1\rangle$ when $p=p(F)<\infty$.

Proposition 2.4. Let $\rho$ be a positive-universal form over $F$. Then any quadratic form $\varphi$ over $F$ with $\operatorname{dim}(\varphi) \geq 2$ is weakly isotropic if and only if $\rho \otimes \varphi$ is isotropic.

Proof. For $m$ sufficiently large $\rho$ is a subform of $m \times\langle 1\rangle$. Therefore, if $\rho \otimes \varphi$ isotropic then $\varphi$ is weakly isotropic. To prove the converse, assume that $\varphi=$ $\left\langle a_{1}, \ldots, a_{n}\right\rangle$ is weakly isotropic. Then there exist $t_{1}, \ldots, t_{n} \in \sum F^{\times 2}$ such that $\widetilde{\varphi}=\left\langle t_{1} a_{1}, \ldots, t_{n} a_{n}\right\rangle$ is isotropic. As the form $\rho$ is positive-universal it represents $t_{1}, \ldots, t_{n}$. Then $\rho \otimes \varphi$ contains $\tilde{\varphi}$ as a subform and is thus isotropic as well.

Corollary 2.5. Let $\varphi$ be a quadratic form of dimension at least 2 over $F$. If $\varphi$ is weakly isotropic, then wi $(\varphi) \leq p(F)$.

Proof. We may assume that $p(F)<\infty$. The statement follows by applying the last proposition to the positive-universal form $p(F) \times\langle 1\rangle$.

The last statement is well-known at least in the case where $F$ is real pythagorean, when it shows that weakly isotropic forms over $F$ are already isotropic and therefore allows us to conclude that the Witt ring $W F$ is torsion-free.

Remark 2.6. We may say that an element $t \in \sum F^{\times 2}$ is positive-universal if the binary form $\langle 1, t\rangle$ has this property, that is, if $D_{F}(\langle 1, t\rangle)=\sum F^{\times 2}$. If $F$ is nonreal, then -1 is a positive-universal element. Over a real field there may exist no positive-universal element. However, if $F$ happens to be a field with finitely many square classes and whose Witt ring $W F$ is of elementary type (cf. [12]), then $F$ has a positive-universal element; this is easily shown by induction on the construction of $W F$ starting from basic Witt rings.

\section{Weakly isotropic subforms}

In this section we give sufficient conditions for a form over $F$ to contain a weakly isotropic subform of dimension at most $m$. In particular, this will give us upper bounds for the invariant $w(F)$. 
The following proposition will be the crucial ingredient in many of the subsequent proofs. It generalizes an observation due to Elman and Lam; in [5, p. 289], they treated the case where the form $\rho$ below is a binary form and obtained in this way their so-called ' $\beta$-decomposition'.

Proposition 3.1. Let $\varphi$ and $\rho$ be quadratic forms over $F$ where $\varphi$ is anisotropic. If $\rho \otimes \varphi$ is isotropic, then $\varphi$ contains a subform $\psi$ such that $\rho \otimes \psi$ is isotropic and $\operatorname{dim}(\psi) \leq \operatorname{dim}(\rho)$.

Proof. Suppose that $\rho \otimes \varphi$ is isotropic. We write $\rho \cong\left\langle b_{1}, \ldots, b_{m}\right\rangle$ with $b_{1}, \ldots b_{m} \in$ $F^{\times}, m=\operatorname{dim}(\rho)$. We consider $\varphi$ as a quadratic map defined on a vector space $V$. Since $\rho \otimes \varphi \cong b_{1} \varphi \perp \cdots \perp b_{m} \varphi$ is isotropic, there exist $x_{1}, \ldots x_{m} \in V$, not all equal to zero, such that $b_{1} \varphi\left(x_{1}\right)+\cdots+b_{m} \varphi\left(x_{m}\right)=0$. Let $W$ we the subspace of $V$ spanned by the vectors $x_{1}, \ldots, x_{m}$. Hence $1 \leq \operatorname{dim}(W) \leq m$. Let $\psi$ denote the restriction of the quadratic map $\varphi$ to this subspace. Since $\varphi$ is anisotropic, so is $\psi$. Hence $\psi$ is a regular quadratic form over $F$ and a subform of $\varphi$. We further have $\operatorname{dim}(\psi) \leq m=\operatorname{dim}(\rho)$ and $\rho \otimes \psi$ is isotropic.

Corollary 3.2. Let $\varphi$ be a quadratic form over $F$ and $m \geq 2$. If $\varphi$ is weakly isotropic of index wi $(\varphi) \leq m$, then there exists a subform $\psi$ of $\varphi$ with $\operatorname{dim}(\psi) \leq m$ such that $\psi$ is weakly isotropic of index wi $(\psi) \leq m$.

Proof. If $\varphi$ is isotropic then we may take for $\psi$ the hyperbolic plane $\langle 1,-1\rangle$. If $\varphi$ is anisotropic, then we put $\rho=m \times\langle 1\rangle$ and apply the proposition.

We are ready to give a first bound on $w(F)$.

Theorem 3.3. If $F$ is real pythagorean, then $w(F)=2$, otherwise $w(F) \leq p(F)$.

Proof. If $F$ is nonreal, then $w(F)=1 \leq p(F)$. Assume for the rest that $F$ is real. If $p(F)=1$, then $F$ is real pythagorean and $\langle 1,-1\rangle$ is the only minimal weakly isotropic form over $F$, in particular $w(F)=2$.

Assume now that $p(F) \geq 2$. Let $\varphi$ be an arbitrary weakly isotropic form over $F$. Then $\operatorname{dim}(\varphi) \geq 2$ as $F$ is real and by $(2.5), \varphi$ has index $w i(\varphi) \leq p(F)$. From (3.2) we obtain that $\varphi$ contains a weakly isotropic subform $\psi$ with $\operatorname{dim}(\psi) \leq p(F)$. This shows that weakly isotropic forms over $F$ of dimension greater than $p(F)$ are not minimal. Hence $w(F) \leq p(F)$.

In view of the theorem it should be mentioned that there cannot be a bound on the Pythagoras number in terms of the $w$-invariant. In fact, in [7] it was shown that for a uniquely ordered field $F$ the Pythagoras number $p(F)$ can be any given positive integer and that $p(F)=\infty$ is also possible. On the other hand, it is obvious that $w(F)=2$ if $F$ is uniquely ordered.

We now obtain a criterion for the existence of weakly isotropic subforms of dimension bounded by a given number.

Proposition 3.4. Let $\varphi$ be an anisotropic quadratic form over $F$ and $m \geq 2$. The following conditions are equivalent: 
(1) $\varphi$ contains a weakly isotropic subform $\psi$ of dimension at most $m$.

(2) There exists an $m$-dimensional totally positive form $\vartheta$ over $F$ such that $\vartheta \otimes \varphi$ is isotropic.

Proof. Assume that $\varphi$ has a weakly isotropic subform $\psi$ with $\operatorname{dim}(\psi) \leq m$. Then taking $\tau=r \times\langle 1\rangle$ for $r$ large enough, we have that $\tau \otimes \psi$ is isotropic. By (3.1), $\tau$ then contains a subform $\vartheta^{\prime}$ with $\operatorname{dim}\left(\vartheta^{\prime}\right) \leq \operatorname{dim}(\psi) \leq m$ such that $\vartheta^{\prime} \otimes \psi$ is isotropic. Then $\vartheta^{\prime}$ is totally positive and $\vartheta^{\prime} \otimes \varphi$ is isotropic. We may further replace $\vartheta^{\prime}$ by $\vartheta=\vartheta^{\prime} \perp\langle 1, \ldots, 1\rangle$ such that $\operatorname{dim}(\vartheta)=m$. This shows that (1) implies (2).

To prove the converse, assume now that $\vartheta$ is a totally positive form over $F$ of dimension $m$ and such that $\vartheta \otimes \varphi$ is isotropic. Then (3.1) says that there exists a subform $\psi$ of $\varphi \operatorname{such}$ that $\operatorname{dim}(\psi) \leq \operatorname{dim}(\vartheta)=m$ and such that $\vartheta \otimes \psi$ is isotropic. Since $\vartheta$ is totally positive, it follows that $\psi$ is weakly isotropic.

Theorem 3.5. Assume that $n \geq 2$ is such that $I^{n} F$ is torsion-free. Then wi $(\varphi) \leq$ $2^{n-2}$ for any weakly isotropic form $\varphi$ of dimension at least 3 over $F$. In particular $w(F) \leq 2^{n-2}$, provided that $n \geq 3$.

Proof. Let $\varphi$ be a quadratic form over $F$ with $\operatorname{dim}(\varphi) \geq 3$. Suppose that $\varphi$ is weakly isotropic. By $(2.2, \mathrm{c})$ there exists a torsion form $\vartheta \in I^{2} F$ which contains $\varphi$ and with $\operatorname{dim}(\vartheta)=2 \operatorname{dim}(\varphi)-2$. Since $I^{n} F$ is torsion-free, $2^{n-2} \times \vartheta$ is hyperbolic. Therefore $\varphi$ has index $w i(\varphi) \leq 2^{n-2}$, by $\left(2.2\right.$, b). If further $n \geq 3$, then $2^{n-2} \geq 2$ and (3.2) yields that $\varphi$ contains a weakly isotropic subform of dimension at most $2^{n-2}$.

Let $u(F)$ denote the $u$-invariant of $F$ as introduced in [5], that is

$$
u(F)=\sup \{\operatorname{dim}(\varphi) \mid \varphi \text { anisotropic torsion form over } F\} .
$$

Corollary 3.6. Assume that $F$ is a real field with $4 \leq u(F)<\infty$ and let $m \geq 2$ be the integer such that $2^{m} \leq u(F)<2^{m+1}$. Then $w(F) \leq 2^{m-1}$, in particular $w(F) \leq \frac{1}{2} u(F)$.

Proof. If $u(F)<2^{m+1}$, then $I^{m+1} F$ is torsion-free, thus the statement follows from (3.5).

To complement these results we give an example showing that the value of the $w$-invariant of a field does neither give a bound on the $u$-invariant nor imply that a certain power of the fundamental ideal in the Witt ring is torsion-free.

Example 3.7. Let $n \in \mathbb{N}$. It is well-known that the field $k=\mathbb{R}\left(\left(t_{1}\right)\right) \ldots\left(\left(t_{n}\right)\right)$ is hereditarily pythagorean (cf. [3, p. 108]), and this fact can be reformulated by saying that for $F=k(X)$ one has $p(F)=2$ (cf. [3, p. 95]). Hence, with (3.3) we obtain that $w(F)=2$. On the other hand, $\left\langle\left\langle 1+X^{2}, t_{1}, \ldots, t_{n}\right\rangle\right\rangle$ is an anisotropic torsion form in $I^{n+1} F$. Therefore $I^{n+1} F$ is not torsion-free and $u(F) \geq 2^{n+1}$. Using that $F(\sqrt{-1})$ is a $\mathcal{C}_{n+1}$-field (cf. [6]), it is not difficult to show that the smallest power of $I F$ which is torsion-free is $I^{n+2} F$ and that $u(F)=u(F(\sqrt{-1}))=2^{n+1}$ (cf. [2, 3.5 Proposition \& 3.6 Remarks]).

Moreover, if for $n \in \mathbb{N}$ we write $F_{n}$ for the field $F$ constructed above with respect to $n$, then $L=\bigcup_{n \in \mathbb{N}} F_{n}$ is a real field with $w(L)=p(L)=2$ and $u(L)=\infty$ and such that $I^{m} L$ is not torsion-free for any $m \in \mathbb{N}$. 


\section{Fields with weak decomposition property}

The following statement gives a criterion to decide whether the invariant $w(F)$ is smaller than a given number.

Theorem 4.1. Assume that $m \geq 3$ is such that there exists no minimal weakly isotropic form of dimension $m$ over $F$. Then $w(F)<m$.

Proof. In order to keep the language simple we shall say that a weakly isotropic form over $F$ is 'small' if its dimension is strictly smaller than $m$. The claim is that every weakly isotropic form over $F$ contains a small weakly isotropic form. We may assume that $F$ is real, so that any weakly isotropic form over $F$ has dimension at least 2.

Since $m \geq 3$, any isotropic form contains a small weakly isotropic form, namely the hyperbolic plane. Using $(2.1, a)$ an arbitrary weakly isotropic form over $F$ can be converted in a finite number of steps into an isotropic form by changing at each step only one coefficient by multiplication with an element of $\sum F^{\times 2}$. It is therefore sufficient to show that the property of having a small weakly isotropic subform is invariant under this operation.

Let $\varphi=\psi \perp\langle a\rangle$ with $a \in F^{\times}$and a form $\psi$ over $F$ and let $\tilde{\varphi}=\psi \perp\langle a t\rangle$ with $t \in \sum F^{\times 2}$. Assuming that $\varphi$ has a small weakly isotropic subform, we want to show that the same is true for $\tilde{\varphi}$. Since $\varphi=\psi \perp\langle a\rangle$ has a small weakly isotropic subform we conclude that $\psi$ has a subform $\gamma$ with $\operatorname{dim}(\gamma)<m$ such that $\gamma \perp\langle a\rangle$ is weakly isotropic. Then $\vartheta=\gamma \perp\langle a t\rangle$ is also weakly isotropic, by (2.1, a). Moreover, $\vartheta$ is contained in $\tilde{\varphi}=\psi \perp\langle a t\rangle$ and $\operatorname{dim}(\vartheta) \leq m$. By the hypothesis on $F$ and $m$, if $\operatorname{dim}(\vartheta)=m$ then the weakly isotropic form $\vartheta$ is not minimal. So in any case $\vartheta$ has a small weakly isotropic subform, which then is also a subform of $\tilde{\varphi}$.

From the theorem we obtain an extension and a different proof of [1, Satz 4], where the implication (4) $\Rightarrow(3)$ is stated.

Corollary 4.2. For a real field $F$, the following conditions are equivalent:

(1) $w(F)=2$.

(2) Every form $\varphi$ over $F$ can be decomposed into $\varphi \cong \gamma \perp \beta_{1} \perp \cdots \perp \beta_{r}$ where $\gamma$ is a strongly anisotropic form and $\beta_{1}, \ldots, \beta_{r}(r \geq 0)$ are torsion binary forms.

(3) Every torsion form $\varphi$ over $F$ can be decomposed into an orthogonal sum of torsion binary forms.

(4) Every torsion 2-fold Pfister form over F contains a torsion binary form.

(5) Every 3-dimensional weakly isotropic form over F contains a torsion binary form.

(6) Every F-quaternion division algebra which is split over every real closure of $F$ contains a totally positive quadratic extension of $F$.

Proof. If $w(F)=2$, then any weakly isotropic form over $F$ contains a weakly isotropic form of dimension 2, hence a torsion binary form. By induction on the dimension it follows that (1) implies (2). The implications (2) $\Rightarrow(3) \Rightarrow(4)$ are obvious. 
The equivalence of (4) and (5) follows from the facts, that any 3-dimensional form $\varphi$ is up to a scalar factor a subform of a unique 2-fold Pfister form $\pi$, that $\varphi$ is weakly isotropic if and only if $\pi$ is torsion, and that $\varphi$ contains a torsion binary form if and only if $\pi$ does. The equivalence of (4) and (6) follows from Pfister's local-global principle together with the one-to-one correspondence between $F$-quaternion (division) algebras and (anisotropic) 2-fold Pfister forms. Finally, (5) implies (1) by the above theorem.

In view of the last corollary, especially of condition (2), we say that a real field $F$ has the weak decomposition property or is a WD-field, if $w(F) \leq 2$.

Examples 4.3. (1) If $I^{3} F$ is torsion-free, then $F$ is a $W D$-field, by (3.5). This shows that any number field as well as any extension of transcendence degree at most two of a real closed field has the weak decomposition property.

(2) If $F$ is an ED-field (cf. [15]), then it is (obviously) a $W D$-field.

(3) By [1, Satz 5], if $F$ is the rational function field $k(X)$ over a number field $k$, then $F$ is a $W D$-field.

(4) If $p(F) \leq 2$, then $F$ is a $W D$-field. In particular the rational function field $F=$ $k(X)$ over any hereditarily pythagorean field $k$ is a $W D$-field, since $p(F)=2$ (cf. [3, p. 95]).

Conjecture 4.4. Let $k$ be a hereditarily pythagorean field. If $F / k$ is a field extension of transcendence degree one, then $F$ is a WD-field.

In view of condition (5) in the corollary above we want to give a characterization of minimality for weakly isotropic forms in dimension three.

Note that in order to study questions about (weak) isotropy we need to know quadratic forms only up to a scalar factor. In particular, when dealing with odddimensional forms we may assume them to have determinant equal to 1 .

Proposition 4.5. Let $\varphi$ be a 3-dimensional quadratic form of trivial determinant over $F$. Let $V$ denote the underlying vector space of $\varphi$.

(a) The form $\varphi$ is weakly isotropic if and only if it is totally indefinite, if and only if for any ordering $P$ of $F$ there exists a non-zero vector $v \in V$ such that $\varphi(v)$ is negative at $P$.

(b) If $\varphi$ is weakly isotropic, then $\varphi$ is minimal weakly isotropic if and only if for any non-zero vector $v \in V$ there exists an ordering $P$ of $F$ such that $\varphi(v)$ is positive at $P$.

Proof. The first equivalence in (a) is well-known and can be checked, for example, by applying Pfister's local-global principle to the form $\langle 1\rangle \perp \varphi$ (which has signature 0 or 4 at any ordering). The second equivalence in (a) follows from the fact that the 3-dimensional form $\varphi$ cannot be negative definite at any ordering of $F$, as its determinant is totally positive.

Assume now that $\varphi$ is weakly isotropic but not minimal. Then it contains a binary subform $\beta$ which is weakly isotropic and therefore of the form $\langle a,-a d\rangle$ where $a \in F^{\times}$and $d \in \sum F^{\times 2}$. Comparing determinants we obtain $\varphi \cong \beta \perp\langle-d\rangle$. 
Hence there exists $v \in V$ such that $\varphi(v)=-d$, which obviously is negative at every ordering of $F$. This shows one implication claimed in (b).

To prove the converse implication, assume that $\varphi$ is weakly isotropic and that there exists $v \in V$ for which $\varphi(v)$ is negative at every ordering of $F$. By the Artin-Schreier Theorem $d=-\varphi(v)$ is a sum of squares in $F$. Decomposing $\varphi$ into $\langle-d\rangle \perp \beta$ and comparing determinants we find a torsion binary form $\beta$ contained in $\varphi$. Hence $\varphi$ is not minimal.

\section{Further results and open problems}

We are going to define three more field invariants. This will allow us to refine the earlier observation that $w(F) \leq p(F)$ holds when $F$ is not real pythagorean.

We denote by $b(F)$ (resp. by $b^{\prime}(F)$ ) the least $m \in \mathbb{N} \cup\{\infty\}$ such that, given any set $S \subset \sum F^{\times 2}$ with $|S|=m+1$ (resp. with $|S|<\infty$ ), there exists an $m$-dimensional totally positive form $\rho$ over $F$ which represents all elements of $S$.

Remarks 5.1. (1) For any integer $m \geq b(F)$ and any $S \subset \sum F^{\times 2}$ with $|S|=m+1$ there exists an $m$-dimensional totally positive form $\rho$ over $F$ which represents all elements of $S$. This is readily shown by induction on $m$. In fact, for $m>b(F)$, writing $S=S^{\prime} \cup\{a\}$ where $\left|S^{\prime}\right|=m$ the induction hypothesis yields that there is a totally positive form $\rho^{\prime}$ of dimension $m-1$ over $F$ representing the elements of $S^{\prime}$, and then one can take $\rho=\rho^{\prime} \perp\langle a\rangle$.

(2) For $m \in \mathbb{N}$, one has $m \geq b(F)$ if and only if for any choice of $m$ elements $t_{1}, \ldots, t_{m} \in \sum F^{\times 2}$ there exists an $m$-dimensional totally positive form $\rho$ over $F$ representing 1 and $t_{1}, \ldots, t_{m}$. Indeed, given a set of $m+1$ elements $\left\{a_{0}, \ldots, a_{m}\right\} \subset \sum F^{\times 2}$, if there is an $m$-dimensional totally positive form $\rho$ over $F$ representing 1 and the products $a_{0} a_{i}$ for $1 \leq i \leq m$, then $a_{0} \rho$ is also totally positive and represents $a_{0}, \ldots, a_{m}$.

This shows in particular that Property B considered in [1] for a real field $F$ corresponds to the condition that $b(F) \leq 2$.

Let $p u(F)$ denote the least integer $m \geq 1$ such that there exists a positiveuniversal form of dimension $m$ over $F$; if there is no positive-universal form over $F$, then let $\operatorname{pu}(F)=\infty$.

Proposition 5.2. For any $F$ one has the inequalities

$$
b(F) \leq b^{\prime}(F) \leq p u(F) \leq p(F) .
$$

Moreover, if any of these numbers is equal to 1 , then $F$ is pythagorean and all four numbers are equal to 1 .

Proof. These facts are obvious from the definitions.

Examples 5.3. (1) If $F$ is a number field, then $b(F)=b^{\prime}(F)=2$. This can be shown by an approximation argument, as it was explained to the author by David Leep.

(2) If $F$ is a real field with finite square class group and such that $W F$ is of elementary type, then in view of (2.6) we obtain $b(F)=b^{\prime}(F)=p u(F) \leq 2$. By the next proposition this implies that $w(F)=2$. 
We obtain the following refinement of (3.3).

Proposition 5.4. If $F$ is not real pythagorean then $w(F) \leq b(F)$.

Proof. By (4.1), it suffices to show that there exists no minimal weakly isotropic form of dimension $n=b(F)+1$. Let $\varphi=\left\langle a_{1}, \ldots, a_{n}\right\rangle$ be a weakly isotropic form over $F$ of dimension $n$. By $\left(2.1\right.$, a), there exist elements $t_{1}, \ldots, t_{n} \in \sum F^{\times 2}$ such that $\tilde{\varphi}=\left\langle a_{1} t_{1}, \ldots, a_{n} t_{n}\right\rangle$ is isotropic. By the definition of $b(F)$, there exists an $(n-1)$-dimensional totally positive form $\rho$ over $F$ representing all the elements $t_{1}, \ldots, t_{n}$. Now, $\rho \otimes \varphi$ contains $\widetilde{\varphi}$ and is therefore isotropic. Hence by (3.1), $\varphi$ contains a weakly isotropic subform of dimension $n-1$.

Proposition 5.5. Assume that $F$ carries a 2-henselian discrete valuation $v$ and let $F_{v}$ be its residue field. Then $w(F)=w\left(F_{v}\right)$.

Proof. Let $t$ be a uniformizer for $v$. Let $\varphi$ be a quadratic form over $F$. We may write $\varphi \cong \varphi_{0} \perp t \varphi_{1}$ where $\varphi_{0}$ and $\varphi_{1}$ have diagonalizations whose entries are units with respect to $v$. It is well-known that $\varphi$ is isotropic (resp. weakly isotropic) over $F$ if and only if at least one of the residue forms $\overline{\varphi_{0}}$ and $\overline{\varphi_{1}}$ is isotropic (resp. weakly isotropic) over $F_{v}$. Therefore $\varphi$ is a minimal weakly isotropic form over $F$ if and only if one of the residue forms is trivial and the other one is a minimal weakly isotropic form over $F_{v}$. This implies the statement.

Remark 5.6. The analogous statement holds for each of the invariants $b, b^{\prime}, p u$ introduced here, as well as for the Pythagoras number. That is, under the hypothesis of the proposition we have $b(F)=b\left(F_{v}\right), b^{\prime}(F)=b^{\prime}\left(F_{v}\right), p u(F)=p u\left(F_{v}\right)$, and $p(F)=p\left(F_{v}\right)$. This follows just from the one-to-one correspondence between the square classes of totally positive elements in $F$ and in $F_{v}$. Note that, in contrast to these invariants, the $w$-invariant is not determined only by the interaction between the totally positive elements of the given field.

By the last proposition, there can be no lower bound on the $w$-invariant in terms of the stability index. The latter field invariant was introduced in [4]. Fields of stability index 0 or 1 are $S A P$-fields (see [16] or [17] for this notion).

Question 5.7. Is there an upper bound on $w(F)$ when $F$ is a $S A P$-field?

Recall that $w(F) \leq 2$ if $F$ is an $E D$-field. This fact motivates to ask:

Question 5.8. What values can $b(F)$ take when $F$ is an $E D$-field?

We have not seen examples of minimal weakly isotropic forms of dimension greater than 3 and thus have no evidence so far that the $w$-invariant can have values larger than 3. Needless to say, the author expects the following.

Conjecture 5.9. Given any number $m \geq 2$, there exists a real field $F$ with a minimal weakly isotropic form of dimension $m$. 
Acknowledgements. The author expresses his gratitude to David Leep and to Jan Van Geel for many discussions, questions, answers, and suggestions. He further gratefully acknowledges the financial support provided by the European RTN Network 'Algebraic K-Theory, Linear Algebraic Groups, and Related Structures' (HPRN-CT-2000-00287) and by the Swiss National Science Foundation (Grant No. 200020-100229/1). Finally, the author thanks the referee and Thomas Unger for very valuable remarks on a preliminary version of this paper.

\section{References}

1. Arason, J.K., Pfister, A.: Zur Theorie der quadratischen Formen über formalreellen Körpern. Math. Z. 153, 289-296 (1977)

2. Becher, K.J.: On fields of $u$-invariant 4. Arch. Math. (Basel) to appear

3. Becker, E.: Hereditarily-Pythagorean fields and orderings of higher level. Monografias de Matemática 29. Instituto de Matemática Pura e Aplicada, Rio de Janeiro, 1978

4. Bröcker, L.: Zur Theorie der quadratischen Formen über formal reellen Körpern. Math. Ann. 210, 233-256 (1974)

5. Elman, R., Lam, T.Y.: Quadratic forms and the $u$-invariant I. Math. Z. 131, 283-304 (1973)

6. Greenberg, M.J.: Lectures on forms in many variables. W. A. Benjamin, Inc., New York, 1969

7. Hoffmann, D.W.: Pythagoras numbers of fields. J. Amer. Math. Soc. 12, 839-848 (1999)

8. Hoffmann, D.W.: Isotropy of quadratic forms and field invariants. Cont. Math. 272, 73-101 (2000)

9. Lam, T.Y.: The algebraic theory of quadratic forms. W. A. Benjamin, Inc., Reading, Mass., 1973

10. Lam, T.Y.: Orderings, valuations, and quadratic forms. CBMS Regional Conf. Ser. in Math. 52, American Mathematical Society, Providence, R.I., 1983

11. Marshall, M.: Some local-global principles for formally real fields. Canad. J. Math. 29, 606-614 (1977)

12. Marshall, M.: The elementary type conjecture in quadratic form theory. Cont. Math. 344, 275-293 (2004)

13. Pfister, A.: Quadratische Formen in beliebigen Körpern. Invent. Math. 1, 116-132 (1966)

14. Pfister, A.: Quadratic Forms with Applications to Algebraic Geometry and Topology. London Math. Soc. Lect. Notes 217, Cambridge University Press, Cambridge, 1995

15. Prestel, A.: Quadratische Semi-Ordnungen und quadratische Formen. Math. Z. 133, 319-342 (1973)

16. Prestel, A.: Lectures on Formally Real Fields. Lecture Notes in Mathematics 1093, Springer, Berlin, 1984

17. Prestel, A., Delzell, Ch.: Positive Polynomials. Springer Monographs in Mathematics. Springer, Berlin, 2001

18. Scharlau, W.: Quadratic and Hermitian forms. Grundlehren der Mathematischen Wissenschaften 270, Springer, Berlin, 1985 